Recent Insights into the Physics of the Sun and Heliosphere:

Highlights from SOHO and Other Space Missions

IAU Symposium, Vol. 203, 2001

P. Brekke, B. Fleck, and J. B. Gurman eds.

\title{
EUV Line Intensities and the Magnetic Field in Solar Active Regions
}

\section{J. Ireland}

ESA at NASA/GSFC, Mailcode 682.3, Building 26, Greenbelt, MD 20771, USA.

\author{
A. Fludra \\ Space Science and Technology Department, Rutherford Appleton \\ Laboratory, Chilton, Didcot, Oxfordshire OX11 OQX, UK.
}

\begin{abstract}
The Coronal Diagnostic Spectrometer (CDS) on SOHO carries out daily synoptic observations of the Sun in four EUV (extreme ultraviolet) spectra: He I $584 \AA$, O V $630 \AA$, Mg IX $368 \AA$ and Fe XVI $360 \AA$, over a 4 arcmin-wide strip along the solar central meridian. Using 53 active regions observed in this data set along with co-temporally observed SOHO-MDI (Michelson Doppler Imager) magnetograms we study the correlation of the chromospheric, transition region and coronal emission with the photospheric magnetic field for meridional active regions, probing the relation between the radiative output and magnetic observables. We also establish empirical, quantitative relations among intensities of different lines, and between intensities and the magnetic field flux.
\end{abstract}

\section{Introduction and analysis method}

Active regions are typically associated with the emergence of magnetic flux through the photosphere. As this flux evolves, the magnetic field spreads and forms loop-like structures that reach up into the corona. Some of these coronal loops become visible as a more intense emission in EUV and X-ray wavelengths and hence outline the field that confines this high temperature plasma. The fact that these loops have an increased emission at coronal temperatures indicates that the electron density and temperatures in these loops is higher here than in the quiet solar corona; it also suggests that some mechanism (or mechanisms) is causing this increased energy deposition in the active region. A wealth of theories exist describing mechanisms that lead to heat deposition in the corona. Rather than looking for evidence for one particular coronal heating mechanism we seek to characterize the global properties of active regions in terms of measured EUV intensity and photospheric line of sight magnetic field. Fludra et al. 1997 presented observations of several active regions and explored a detailed spatial relationship between the hot active region loops (1-2 MK), seen in coronal lines, and the cooler loops, seen in the transition region and chromospheric lines $(20,000-400,000 \mathrm{~K})$. 
In this paper, we examine the emission in 4 EUV lines (He I $584.33 \AA$, O $\mathrm{V} 629.73 \AA, \mathrm{Mg}$ IX $368.06 \AA$ and Fe XVI $360.76 \AA$ ) for fifty three active regions identified in CDS synoptic data from May 1996 to June 1998, and study the statistical relationship between the active region intensities of these spectral lines and the corresponding relevant magnetic field flux. This extends the study of Schrijver et al, 1985 and Schrijver, 1987 to a larger sample of active regions and different EUV lines. The CDS synoptic dataset (run every day from about 00:00 - 06:30 UT) consists of a strip of 9 overlapping $240 \times 240 \mathrm{arcsec}^{2}$ rasters extending from the north pole to the south pole down the central meridian. These emission spectra provide a map of the solar atmosphere from the chromosphere through to the corona. Synoptic MDI full disk magnetograms closest to the CDS observation time are used to supply the photospheric magnetic information relevant to the active region.

The contours used (see Figure 1 for an example active region) to discriminate between active and non-active region in the CDS emission lines are defined as follows: $\mathrm{He} \mathrm{I}, \min \left(\bar{i}+2 \sigma_{i}, 4000\right), \mathrm{O} \mathrm{V}, \bar{i}+2 \sigma_{i}, \mathrm{Mg} \mathrm{IX}, \min \left(\bar{i}+2 \sigma_{i}, 900\right)$ and $\mathrm{Fe} \mathrm{XVI}, \min \left(\bar{i}+\sigma_{i}, 500\right)$ where $\bar{i}$ is the image average, and $\sigma_{i}$ is the image standard deviation (in units of ergs $/ \mathrm{cm}^{2} / \mathrm{sec} / \mathrm{sterad}$ ). It is found that using a simple $\bar{i}+n \sigma_{i}$ (for $n=1$ or 2 ) prescription excludes too much bright material and so a ceiling is assigned in order to properly include bright emission The exception to this is $\mathrm{O} \mathrm{V}$, where taking $n=2$ includes all the bright material very well.

Emission inside these contours is assumed to be active region related: all emission outside these contours is assumed to be unrelated to the active region. In MDI magnetograms, we associate the magnetic field having an absolute value of 50 Gauss or more with the active region. The total EUV line intensity is defined as $I=\sum_{A} I_{x y}$ where $I_{x y}$ is the intensity at $(x, y)$ in bright intensity area $A$. The average intensity $\bar{I}$ is $I$ divided by the number of pixels in $A$. Similarly, we also define $\phi=\sum_{M}\left|B_{x y}\right|$ as the total unsigned magnetic flux where $M$ is the area of strong line of sight photospheric flux. Again, the average is found by dividing by the number of pixels. Fisher et al, 1998 find that soft X-ray emission is best correlated with $\phi$; it is used here to correlate EUV fluxes. Porter \& Klimchuk, 1995 also find a strong correlation between soft X-ray emission and $\phi$. Log-log scatter plots of $I-I, \phi-I$ (see Figure 2), $\bar{I}-\bar{I}$ and $\bar{\phi}-\bar{I}$ for differing lines are formed. The exponent of a $X^{p}$ power law fitted to the scatter and the Spearman rank correlation $\rho$ are calculated and are shown in Tables 1 and 2.

\section{Results and Conclusions}

The degree of correlation between one emission line and another varies from relatively high to high across all line pairs in total intensity. Once we remove the effect of area (i.e., in the $\bar{I}-\bar{I}$ intensity results) the high degree of correlation disappears (we assume $|\rho|<0.5$ indicates a poor correlation). This indicates that the total area of the active region plays a significant role in determining the correlation between the emission in one line and another, extending the conclusion of Schrijver et al, 1985. in the lines Lyman $\alpha 1216 \AA$, O VI $1032 \AA$ and $\mathrm{Mg} X 625 \AA$. Total unsigned magnetic flux appears to be relatively well to very well correlated with the total EUV line emission in all 4 lines. Note also that the coronal Fe XVI total emission is also well correlated with $\phi$. The correlations of 
$\bar{\phi}$ with $\bar{I}$ are all slightly lower than their 'total' counterparts, excepting $\bar{\phi}$ versus $\bar{I}(\mathrm{Mg} \mathrm{IX})$, which is very much smaller than the total equivalent.

In conclusion, very good correlations amongst the total EUV line intensities have been found. In addition, there are very good correlations between the total EUV line intensities and the total unsigned magnetic flux. Total active region quantities $I$ and $\phi$ are better correlated than their average counterparts, extending the results on soft $\mathrm{X}$-ray $/ \phi$ correlations to cooler temperatures; however, note that good correlations still exist between $\bar{I}$ and $\bar{\phi}$. Most of the variability in $I$ and $\phi$ is due to active region size. Various heating mechanisms will be examined in further work to explain the observed dependencies.

Table 1. $I-I$ and $I-\phi$ results. Each entry in the table consists of 2 numbers: the first is the best fit power law exponent $p$, the second is the Spearman rank correlation coefficient $\rho$ The table headers indicate the independent variable $X$ whilst the leftmost column indicates the dependant variable. See section 1 for more detail.

\begin{tabular}{lcccc} 
& $I\{\mathrm{He} \mathrm{I}\}$ & $I\{\mathrm{Mg} \mathrm{IX}\}$ & $I\{\mathrm{Fe} \mathrm{XVI}\}$ & $\phi$ \\
\hline$I\{\mathrm{He} \mathrm{I}\}$ & - & - & - & $0.80[0.95]$ \\
$I\{\mathrm{O} \mathrm{V}\}$ & $0.57[0.89]$ & $0.48[0.78]$ & $0.31[0.85]$ & $0.44[0.80]$ \\
$I\{\mathrm{Mg} \mathrm{IX}\}$ & $0.81[0.77]$ & - & $0.50[0.77]$ & $0.72[0.79]$ \\
$I\{\mathrm{Fe} \mathrm{XVI}\}$ & $1.59[0.97]$ & - & - & $1.39[0.96]$ \\
\hline \hline
\end{tabular}

Table 2. $\quad \bar{I}-\bar{I}$ and $\bar{I}-\bar{\phi}$ results. Layout is the same as Table 1 .

\begin{tabular}{lcccc} 
& $\bar{I}\{\mathrm{He} \mathrm{I}\}$ & $\bar{I}\{\mathrm{Mg}$ IX $\}$ & $\bar{I}\{\mathrm{Fe} \mathrm{XVI}\}$ & $\bar{\phi}$ \\
\hline $\bar{I}\{\mathrm{He} \mathrm{I}\}$ & - & - & - & $1.98[0.90]$ \\
$\bar{I}\{\mathrm{O} \mathrm{V}\}$ & $0.10[0.24]$ & $-1.02[-0.42]$ & $-0.39[-0.49]$ & $1.56[0.76]$ \\
$\bar{I}\{\mathrm{Mg} \mathrm{IX}\}$ & $0.79[0.37]$ & - & $0.10[-0.01]$ & $0.60[0.49]$ \\
$\bar{I}\{\mathrm{Fe} \mathrm{XVI}\}$ & $2.61[0.91]$ & - & - & $2.41[0.87]$ \\
\hline \hline
\end{tabular}

\section{References}

Fludra, A., Brekke, P., Harrison, R. A., Mason, H. E., Pike, C. D., Thompson, W. T., \& Young, P. R., 1997, Sol. Phys., 175, 487

Fisher, G. H., Longcope, D. W., Metcalf, T. R. \& Pevtsov, A. A., 1998, ApJ, 508,885

Porter, L. J., \& Klimchuk, J. A., 1995, ApJ, 454, 499

Schrijver, C. J., 1987, A\&A, 172, 111

Schrijver, C. J., Zwaan, Maxson, C. W., \& Noyes, R. W., 1985, A\&A, 149, 123

Acknowledgments. JI would like to thank the ESA External Fellowship program and the European Union "Solar Magnetometry Network" for their support. 


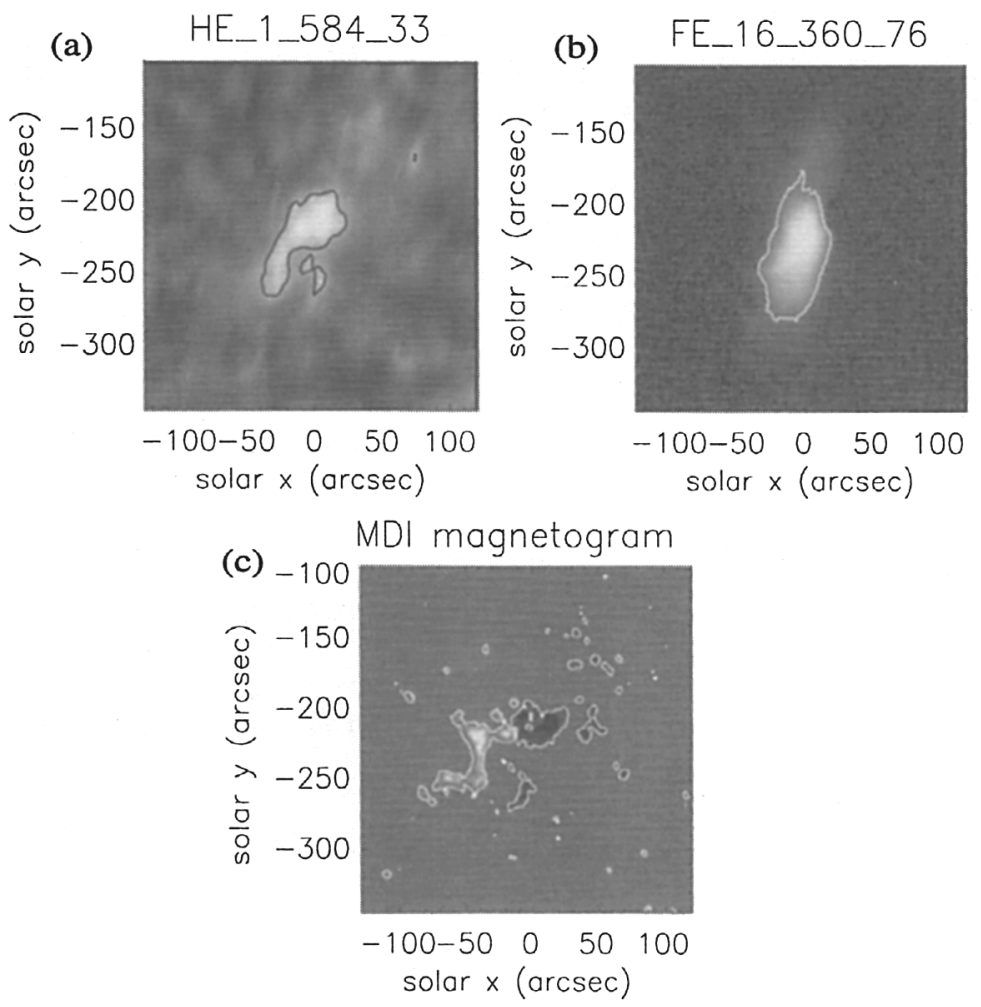

Figure 1. Example active region in two CDS lines (a,b) with corresponding magnetogram (c). Contours enclose bright emission/magnetic flux we assume to be associated with the active region.
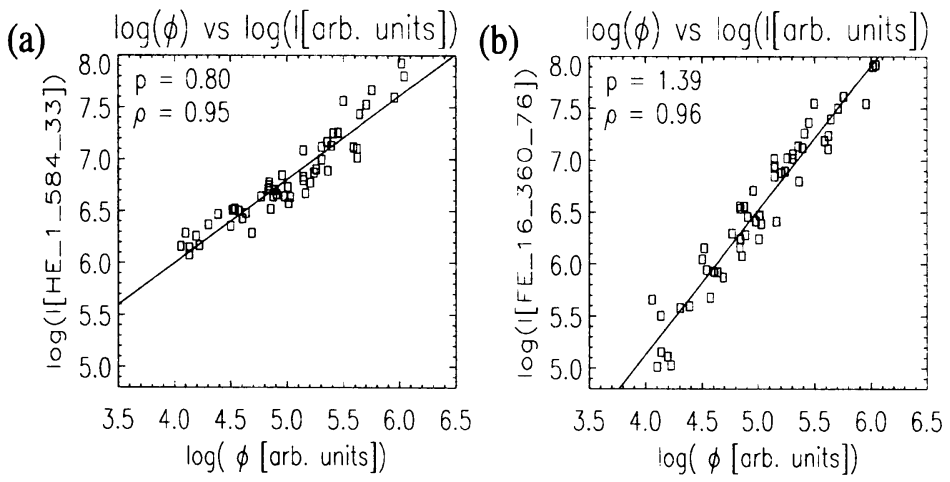

Figure 2. Plots of total unsigned magnetic flux versus total EUV line intensity in arbitrary units. Also quoted is the best fit power law power $p$ and Spearman rank correlation coefficient $\rho$. 